\title{
4-Octyl Itaconate Activates Nrf2 Signaling to Inhibit Pro-Inflammatory Cytokine Production in Peripheral Blood Mononuclear Cells of Systemic Lupus Erythematosus Patients
}

\author{
Chun Tang $^{\mathrm{a}}$ Xiaohua Wang ${ }^{\mathrm{a}}$ Yingying Xie $^{\mathrm{a}}$ Xiaoyan Cai ${ }^{\mathrm{b}} \quad \mathrm{Na} \mathrm{Yu}{ }^{\mathrm{b}}$ \\ Yudan $\mathrm{Hu}^{\mathrm{a}}$ Zhihua Zheng ${ }^{\mathrm{a}}$ \\ aDepartment of Nephrology, Kidney and Urology Center, the Seventh Affiliated Hospital of Sun Yat-sen \\ University, Shenzhen, 'Department of Rheumatology, Guangzhou First People's Hospital, The Second \\ Affiliated Hospital of South China University of Technology, Guangzhou, China
}

\section{Key Words}

SLE $•$ PBMCs $・ 4$-octyl itaconate $・$ Nrf2 signaling $\bullet$ Pro-inflammatory cytokines

\begin{abstract}
Background/Aims: Increased production of multiple pro-inflammatory cytokines, including tumor necrosis factor (TNF)- $\alpha$, interleukin (IL)-1 $\beta$, and IL- 6 , plays an essential pathogenic role in the progression of systemic lupus erythematosus (SLE). Recent studies have characterized itaconate as a novel and potent nuclear-factor-E2-related factor 2 (Nrf2) activator that activates Nrf2 signaling by alkylating cysteine residues on Keap1 (Kelch-like ECH-associated protein 1). Methods: THP-1 human macrophages and peripheral blood mononuclear cells (PBMCs) of SLE patients were treated with 4-octyl itaconate (OI). Nrf2 signaling activation was tested by qPCR assay and western blotting. mRNA expression and the production of multiple pro-inflammatory cytokines were tested by qPCR and enzyme-linked immunosorbent assays, respectively. Nuclear factor (NF)-KB activation was tested by the p65 DNA-binding assay. Results: We demonstrated that OI, the cell-permeable derivative of itaconate, induced Keap1-Nrf2 dissociation, Nrf2 protein accumulation, and nuclear translocation, which enabled the transcription and expression of multiple Nrf2-dependentantioxidant enzymes (heme oxygenase-1, $\mathrm{NAD}(\mathrm{P}) \mathrm{H}$ :quinone oxidoreductase 1 , and glutamate-cysteine ligase modifier subunit) in THP-1 human macrophages. OI also induced significant Nrf2 activation in SLE patient-derived PBMCs. OI pretreatment inhibited mRNA expression and the production of multiple pro-inflammatory cytokines (TNF- $\alpha$, IL-1 $\beta$, and IL-6) in SLE patient-derived PBMCs and lipopolysaccharide (LPS)-activated THP-1 cells. Ol potently inhibited NF-KB activation in SLE patient-derived PBMCs and LPS-activated THP-1 cells. Importantly, Nrf2 silencing (by targeted

C. Tang, $X$. Wang, and Y. Xie contributed equally to this work.

Dr. Zhihua Zheng

and Dr. Chun Tang




\section{Cellular Physiology Cell Physiol Biochem 2018;51:979-990

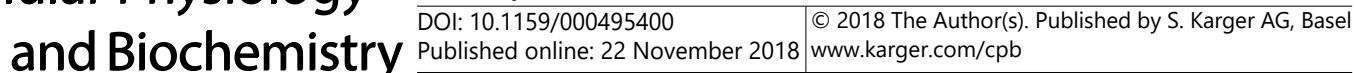 \\ Tang et al.: 4-Octyl Itaconate Activates Nrf2 Signaling in Monocytes}

short hairpin RNA) or knockout (by CRISPR/Cas9 gene-editing method) almost abolished OIinduced anti-oxidant and anti-inflammatory actions in SLE patient-derived PBMCs and LPSactivated THP-1 cells. Conclusion: Ol activates Nrf2 signaling to inhibit the production of pro-inflammatory cytokines in human macrophages and SLE patient-derived PBMCs. OI and itaconate could have important therapeutic value for the treatment of SLE.

(C) 2018 The Author(s)

Published by S. Karger AG, Basel

\section{Introduction}

Systemic lupus erythematosus (SLE) is a multifactorial autoimmune disease [1-3]. It is characterized by inflammation in different organ systems and immunological abnormalities, including the presence of auto-reactive $T$ cells and hyperactive $B$ cells [1-3]. In the past few decades, significant achievements have been made in gaining an understanding of the pathobiological mechanisms of SLE, by identifying genetic variants via mouse models, gene expression studies, and epigenetic analyses [1-3]. However, the prognosis for severe SLE is not satisfactory, possibly due to the lack of effective and targeted treatments [1-3].

Dysregulation of pro- and anti-inflammatory cytokines is essential for the regulation of systemic inflammation, local tissue damage, and immunoreactivity in the progression of SLE $[4,5]$. Abnormal production of multiple pro-inflammatory cytokines has been detected in SLE patients $[4,5]$. Elevations of cytokines, including tumor necrosis factor (TNF)- $\alpha$, interleukin (IL)-1 $\beta$, and IL-6, play important pathogenic roles in SLE. Conversely, inhibition of these cytokines could significantly slow the progression of SLE [5-9].

The transcription factor nuclear-factor-E2-related factor 2(Nrf2, also known asNFE2L2) mediates the transcription and expression of many antioxidant and anti-inflammatory genes [10-12]. Nrf2 deficiency leads to the development of lupus-like autoimmune nephritis in aged female mice, indicating that Nrf2 is vital to the development and progression of SLE [13]. Nonactivated Nrf2 remains in the cytoplasm by binding to its suppressor protein Kelch-like ECHassociated protein 1 (Keap1) [10-12]. Keap1-Nrf2 association dictates Nrf2 ubiquitination and proteasomal degradation in the Cul3-ubiquitin ligase complex [10-12]. Once activated, Nrf2 will separate from Keap1, enabling Nrf2 protein stabilization, accumulation, and translocation to cell nuclei [10-12]. Nrf2 will then bind to antioxidant responsive element (ARE) [10-12], initiating the transcription of multiple antioxidant, anti-inflammatory, and detoxifying enzymes, including heme oxygenase-1 (HO-1), NAD(P)H:quinone oxidoreductase 1 (NQO1), $\gamma$-glutamyl cysteine ligase catalytic subunit, and a modifier subunit (GCLM) [10], leading to a significant antioxidant response $[14,15]$. Furthermore, activation of Nrf2 signaling could also exert a potent anti-inflammatory effect by inhibiting nuclear factor (NF)- $\kappa$ B activation [14].

A recent study by Mills et al. demonstrated that itaconate is a novel activator of Nrf2 [16]. Itaconate directly alkylates cysteine residues 151, 257, 288, 273, and 297 on Keap1, causing Nrf2 protein stabilization, accumulation, and activation [16]. Additionally, a new cell-permeable itaconate derivative, 4-octyl itaconate (OI), increases the expression of Nrf2 downstream genes with antioxidant and anti-inflammatory capacities [16]. The current study tested the potential effect of OI on SLE patient-derived peripheral blood mononuclear cells (PBMCs). We will show that OI activates Nrf2 signaling to efficiently inhibit the production of pro-inflammatory cytokines in PBMCs.

\section{Materials and Methods}

Chemicals, reagents and antibodies

OI was synthesized by Min-de Biotech (Suzhou, China) based on a previously described protocol [16]. Lipopolysaccharide (LPS) and puromycin were purchased from Sigma-Aldrich Co. (St. Louis, MO). Antibodies were obtained from Cell Signaling Technology (Danvers, MA) and Santa Cruz Biotechnology Inc. (Santa Cruz, CA). Fetal bovine serum (FBS), Dulbecco's modified Eagle's medium (DMEM)/Rosswell 


\section{Cellular Physiology Cell Physiol Biochem 2018;51:979-990

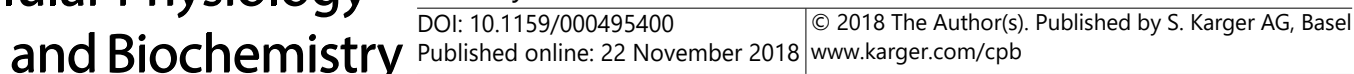 \\ Tang et al.: 4-Octyl Itaconate Activates Nrf2 Signaling in Monocytes}

Park Memorial Institute (RPMI) medium, antibiotics, and all other cell culture reagents were obtained from Hyclone Co. (Logan, UT). Cell lysis buffer was provided by Beyotime Biotechnology (Wuxi, China). TRIzol reagents were purchased from GibcoBRL (Gaithersburg, MD). mRNA primers and all other sequences were synthesized by GenePharma Co. (Shanghai, China).

THP-1 cell culture

Established THP-1 human macrophages $[17,18]$ were provided by the Cell Bank of Shanghai Institute of Biological Science (Shanghai, China). THP-1 macrophages were cultured in RPMI- 1640 medium with $10 \%$ FBS.

\section{Ex-vivo culture of PBMCs}

As previously described [19], PBMCs from nine SLE patients were collected via the lymphocyte separation medium (Sigma-Aldrich). Whole blood $(30 \mathrm{~mL}$ ) was layered on $20 \mathrm{~mL}$ Lymphoprep and spun for $20 \mathrm{~min}$ at 2, $000 \mathrm{rpm}$. PBMCs were isolated from the middle layer. The primary PBMCs were cultured in FBS-containing DMEM medium and essential supplements [20]. The protocols using human cells were approved by the ethics committees of the Guangzhou First People's Hospital. Written-informed consent was acquired from each participant.

\section{Quantitative real-time reverse transcription PCR assay}

Total cellular RNA was extracted by the TRIzol reagents. Reverse transcription was performed using the ReverTra Ace qPCR RT kit (Toyobo, Tokyo, Japan). The ABI Prism 7700 Real-Time PCR system (Applied Biosystems, Foster City, CA) was applied for the quantitative real-time reverse transcription PCR (qPCR) assay to analyze $500 \mathrm{ng}$ cDNA from each sample. The mRNA primers for $I L-1 \beta, I L-6, T N F-\alpha$, and glyceraldehyde-3-phosphate dehydrogenase (GAPDH) were based on a previous study [21]. mRNA primers for HO1, NQO1, and GCLM were provided by Dr. Huang [22]. Melting curve analysis was applied to calculate the product melting temperature [23]. We utilized the $2^{-\Delta \Delta c t}$ method for mRNA quantification, using GAPDH as the reference gene.

\section{NQO1 activity assay}

The detailed protocol has been described previously [16]. In brief, the inducer potency was quantified by use of the NQO1 bioassay. Cells ( $10^{4}$ per well of a 96-well plate) were grown for $24 \mathrm{~h}$. After the treatment, NQ01 enzyme activity was quantified in cell lysates using menadione as the substrate.

Cell death assay

After the treatments, cell death was tested by trypan blue staining; cells positive for trypan blue staining were considered dead [24] and the ratio of live/dead cells was recorded. Lactate dehydrogenase (LDH) release into the culture medium was tested for the quantitative measurement of cell death. A twostep LDH assay kit (Takara, Tokyo, Japan) [25] was utilized to examine the LDH content in the medium [25, 26], which was normalized to the total LDH.

\section{Western blotting}

Following the treatments, $30 \mu \mathrm{g}$ of protein lysates were separated in a denaturing $10 \%$ polyacrylamide gel and transferred to a polyvinylidene difluoride (PVDF; $0.22 \mu \mathrm{m}$ ) membrane (Millipore, Shanghai, China). After several washes in Tris-buffered saline with Tween 20, PVDF blots were incubated with primary and secondary antibodies, which were then detected by enhanced chemiluminescence (ECL) reagents (Amersham). Image software (National Institutes of Health, Bethesda, MD) was utilized for the quantification of the total gray of each protein band, and the values were normalized to the loading control.

Enzyme-linked immunosorbent assay assay of cytokines

THP-1 cells or the primary human PBMCs were seeded to the 24-well tissue culture plates at a density of $0.5 \times 10^{5}$ cells/well. Following treatment, supernatants were collected and cytokine concentrations were determined using IL-1 $\beta$, IL-6, and TNF- $\alpha$ kits from BD OptEIA (BD Biosciences, San Jose, CA). 


\section{Cellular Physiology Cell Physiol Biochem 2018;51:979-990 \begin{tabular}{l|l|l} 
and Biochemistry & Dublished online: 22 November 2018 & $\begin{array}{l}\text { (c) } 2018 \text { The Author(s). Published by S. Karger AG, Basel } \\
\text { www.karger.com/cpb }\end{array}$
\end{tabular} \\ Tang et al.: 4-Octyl Itaconate Activates Nrf2 Signaling in Monocytes}

\section{$N F-\kappa B(p 65)$ DNA-binding activity}

The nuclear protein lysates were extracted by the commercially available nuclei-extraction reagents from Sigma-Aldrich. The TransAM ${ }^{\mathrm{TM}}$ enzyme-linked immunosorbent assay (ELISA) kit (Active Motif, Carlsbad, CA) was then applied to examine NF- $\mathrm{kB}$ (p65) DNA-binding activity, according to the manufacturer's protocol. For each treatment, $2.5 \mu \mathrm{g}$ of nuclear extracts were placed in a 96-well plate for binding to the immobilized p65 consensus sequence, and primary and secondary antibodies were added. After the colorimetric reaction, the OD value was measured in an ELISA reader at $450 \mathrm{~nm}$.

\section{Co-Immunoprecipitation assay}

For each treatment, $800 \mu \mathrm{g}$ of total cell lysates were pre-cleared by the protein A/G Sepharose (SigmaAldrich). Thereafter, the lysates were incubated with anti-Keap1 antibody (Santa Cruz Biotechnology) overnight. Protein A/G Sepharose (30 $\mu \mathrm{L}$ for each treatment) was then added. Keap 1 co-immunoprecipitation with Nrf2 was tested via Western blotting.

\section{Lipid peroxidation assay}

Using a previously described method [27], cellular lipid peroxidation was tested via TBAR (thiobarbituric acid reactive substances) activity [28]. Briefly, $30 \mu \mathrm{g}$ of total cell lysates per treatment were mixed with a solution of $20 \%$ of acetic acid and thiobarbituric acid. After heating, the mixture was centrifuged, and the red pigment dye in the supernatant was examined by a microplate reader [28].

\section{Nrf2 short hairpin RNA}

Lentiviral human Nrf2 short hairpin RNA (shRNA) was purchased from Santa Cruz Biotechnology (sc37030-V) [29]. Nrf2 shRNA lentivirus (20 $\mu \mathrm{L}$ virus/mL medium) was added directly to THP-1 cells for 36 h. Thereafter, puromycin $(5.0 \mu \mathrm{g} / \mathrm{mL})$ was added to select stable cells for 12 days. Nrf2 knockdown in the stable cells was verified by Western blotting. A knockdown efficiency over $90 \%$ was acceptable. For the primary PBMCs, Nrf2 shRNA lentivirus (20 $\mathrm{L}$ virus/mL medium) was added for $36 \mathrm{~h}$ with no puromycin selection.

\section{Nrf2 knockout}

Lenti-CRISPR-green fluorescent protein (GFP)-Nrf2 knockout (KO) construct was provided by Dr. Li [30]. The construct was transfected to THP-1 cells using Lipofectamine 2000 (Invitrogen, Carlsbad, CA). GFP-positive cells were sorted by fluorescence-activated cell sorting, and single cells were further cultured in 96-well plate to generate monoclonal stable cells. Nrf2 KO was confirmed by Western blotting and qPCR.

\section{Statistics analysis}

Data were expressed as the mean \pm standard deviation. Statistical analysis was performed by SPSS software (version 21.0, SPSS Inc., Chicago, IL) and $p<0.05$ was regarded as statistically significant. For comparisons among multiple groups, two-way ANOVA with the Bonferroni post hoc test was performed. The two-tailed unpaired T test (Excel 2013) was applied to test the significance of the difference between two treatment groups.

\section{Results}

OI activated Nrf2 signaling in THP-1 cells and SLE patient-derived PBMCs

We first tested the potential effect of OI, the cell-permeable derivative of itaconate [16], on Nrf2 signaling in THP-1 human macrophages [31, 32]. By performing the coimmunoprecipitation assay, we showed that treatment with OI $(25 \mu \mathrm{M}, 2 \mathrm{~h})$ disrupted the association between Keap1-Nrf2 in THP-1 cells (Fig. 1A), which was followed by Nrf2 protein stabilization and accumulation in the cytoplasm (Fig. 1B). By analyzing the nuclear proteins, we showed that OI-stabilized Nrf2 translocated to cell nuclei (Fig. 1C), which is a key step for Nrf2 activation [10,33]. Indeed, we showed that mRNA (Fig. 1D) and protein (Fig. 1E) expression of Nrf2-dependent genes, including HO1 (or Hmox1), NQO1, and GCLM, were significantly increased in OI-treated THP-1 cells. Furthermore, the activity of NQO1 


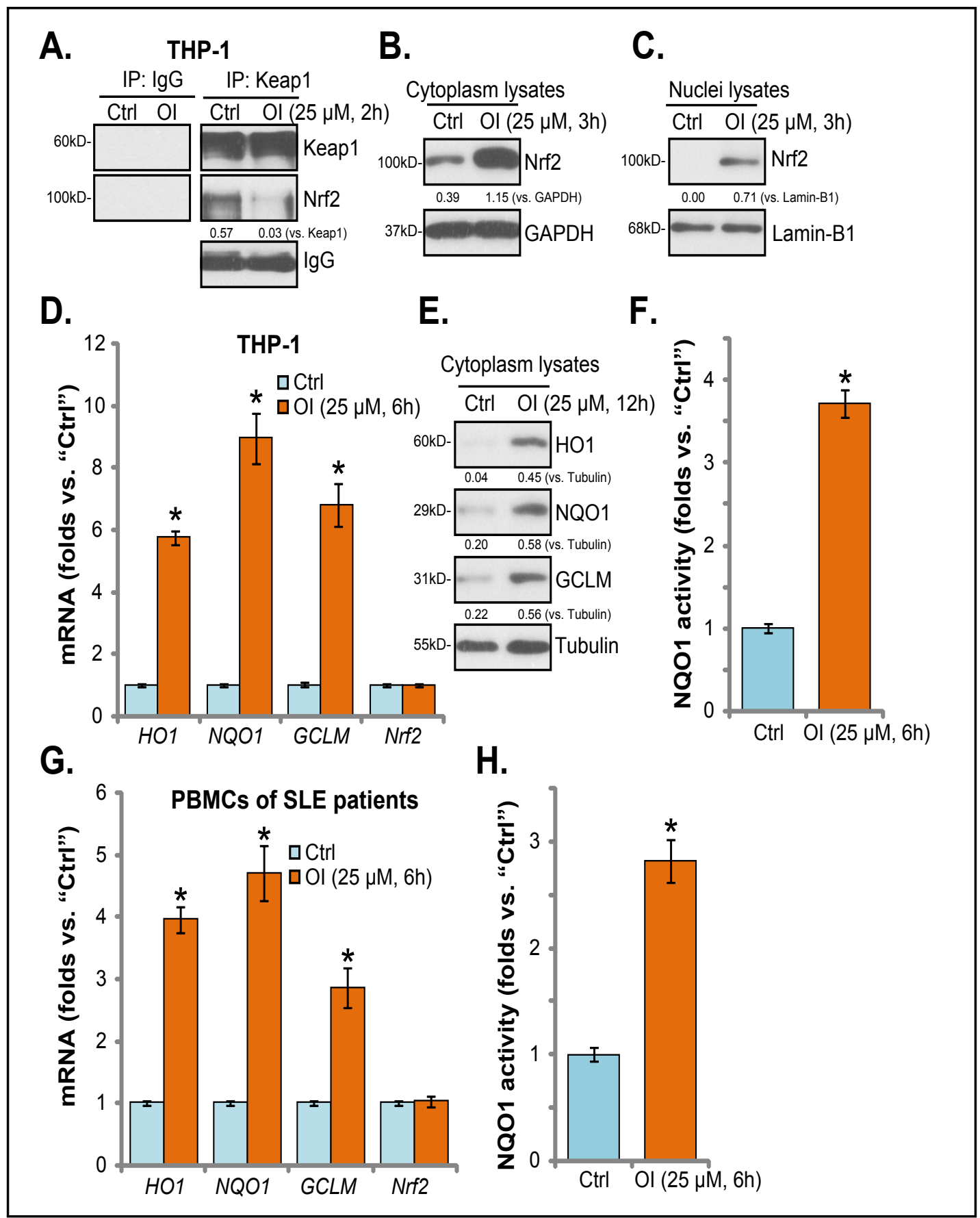

Fig. 1. OI activated Nrf2 signaling in THP-1 cells and SLE patient-derived PBMCs. THP-1 cells (A-F) and SLE patient-derived PBMCs (G and H) were treated with OI $(25 \mu \mathrm{M})$ or vehicle control (Ctrl) for the indicated times. Keap1-Nrf2 association was tested by the co-immunoprecipitation assay (A). mRNA and protein expression levels of the listed genes were tested by qPCR assay (D and G) and Western blotting (analyzing both the cytoplasm and nuclei lysates, B, C, and E), respectively. Relative NQO1 activity was also tested (F and $\mathrm{H}$ ). Expression of the listed proteins was quantified and normalized to the loading control (B, C, and E). Data are presented as the mean \pm standard deviation. In D and F, each assay was replicated five times (five replicate well/dishes). ${ }^{*} \mathrm{p}<0.05$ vs. Ctrl cells. The experiments in this figure were repeated three times, and similar results were obtained. 
A.

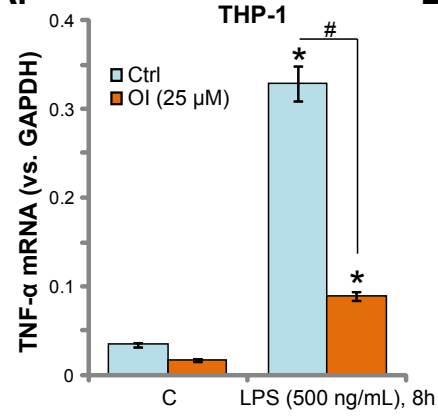

D.

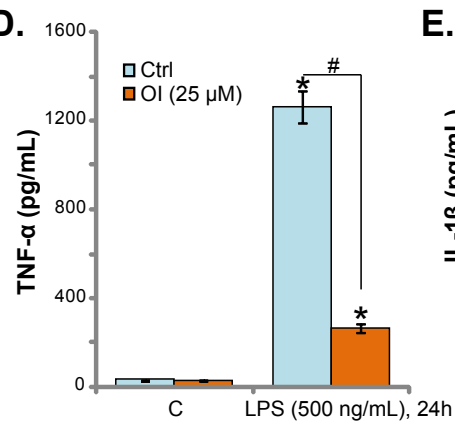

B.

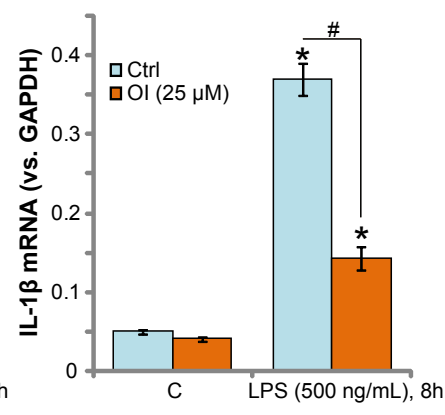

C.

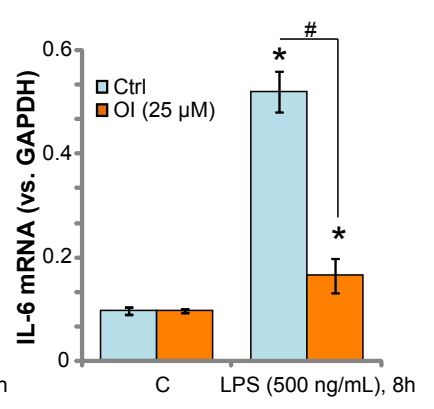

F.
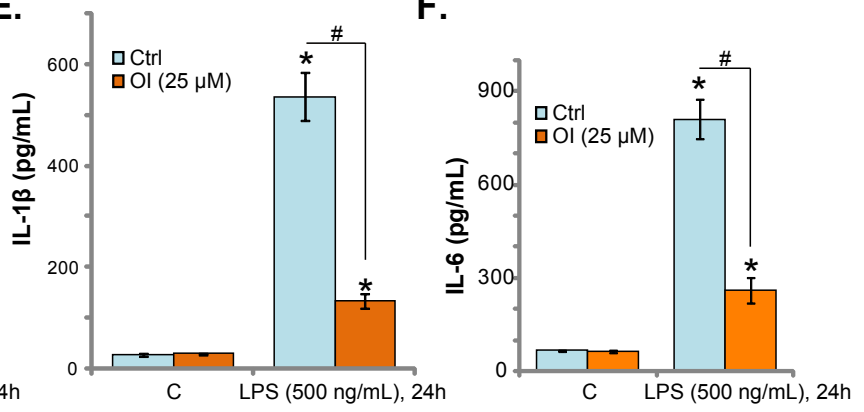

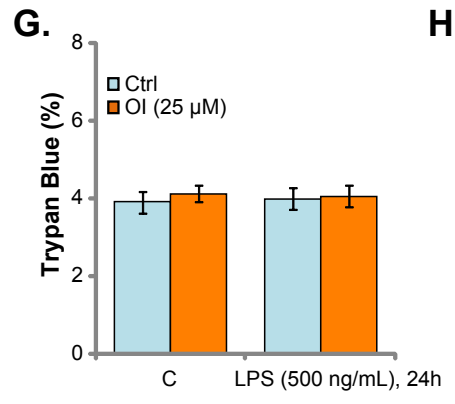

I.

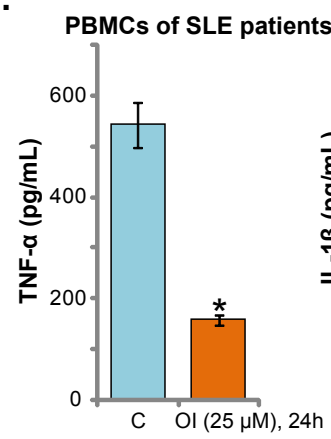

J.

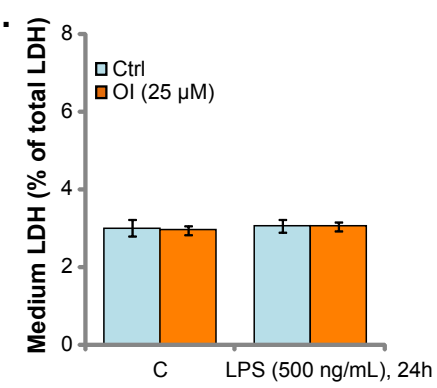

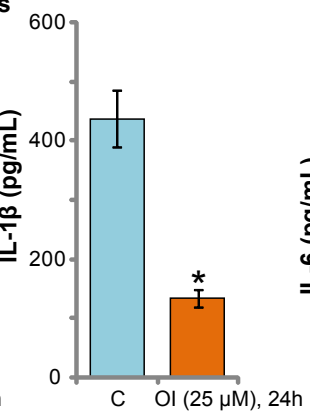

K.

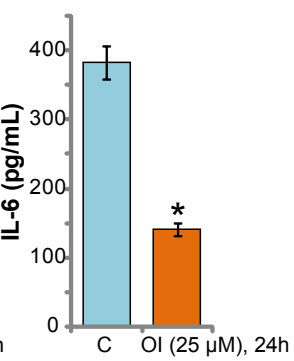

L.

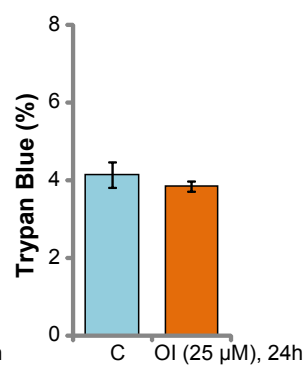

Fig. 2. OI inhibited the production of pro-inflammatory cytokines in LPS-treated THP-1 cells and SLE patient-derived PBMCs. THP-1 cells were pre-treated with OI $(25 \mu \mathrm{M})$ for $60 \mathrm{~min}$, followed by LPS (500 $\mathrm{ng} / \mathrm{mL}$ ) stimulation for the indicated times. TNF- $\alpha$, IL-1 $\beta$, and IL- 6 mRNA (A-C) and protein levels in the medium (D-F) were tested by qPCR assay and ELISA assay, respectively. Cell death was tested by Trypan blue staining assay (G) and LDH release assay (H). SLE patient-derived PBMCs were treated with OI ( $25 \mu \mathrm{M})$ for $24 \mathrm{~h}$; TNF- $\alpha(\mathrm{I}), \mathrm{IL}-1 \beta(\mathrm{J})$, and IL-6 (K) protein levels in the medium are shown. Cell death was tested by trypan blue staining assay (L). Data are presented as the mean \pm standard deviation. In $\mathrm{A}-\mathrm{H}$, the assays were replicated five times (five replicate well/dishes). I-L show the integrated results of PBMCs from all SLE patients $(n=5)$. Ctrl indicates vehicle control. C indicates untreated control cells. ${ }^{*} \mathrm{p}<0.05$ vs. $C$ treatment. ${ }^{\#} \mathrm{p}<0.05$. The experiments in this figure were repeated three times, and each time the results were similar. 


\section{Cellular Physiology Cell Physiol Biochem 2018;51:979-990 and Biochemistry DOl: 10.1159/000495400 2018 O 2018 The Author(s). Published by S. Karger AG, Basel

was boosted after OI treatment (Fig. 1F). However, the Nrf2 mRNA level was unchanged by OI treatment (Fig. 1D). These results suggest that OI disrupts the KEAP1-Nrf2 association to activate the Nrf2 signaling cascade in THP-1 human macrophages. Importantly, in the SLE patient-derived PBMCs, OI treatment $(25 \mu \mathrm{M})$ similarly boosted mRNA expression of HO1, NQO1 and GCLM, but not Nrf2 genes (Fig. 1G; integrated results of nine patients). A significant increase in NQ01 activity was also detected in OI-treated PBMCs (Fig. 1H). These results together show that OI activates Nrf2 signaling in THP-1 cells and SLE patient-derived PBMCs.

OI inhibited pro-inflammatory cytokine production in LPS-treated THP-1 cells and SLE patient-derived PBMCS

One primary aim of this study was to test the potential effect of itaconate on the production of pro-inflammatory cytokines. THP-1 human macrophages were treated with LPS (500 ng/mL), which induced the transcription of TNF- $\alpha$ (Fig. 2A), IL-1 $\beta$ (Fig. 2B), and $I L-6$ mRNA (Fig. 2C). Pre-treatment (for $60 \mathrm{~min})$ with OI $(25 \mu \mathrm{M})$ largely inhibited LPS-induced TNF- $\alpha, I L-1 \beta$, and $I L-6$ mRNA expression (Fig. 2A-C). In addition, LPS-induced production of TNF- $\alpha$, IL-1 $\beta$, and IL- 6 proteins was also largely inhibited by OI pre-treatment (Fig. 2D-F). These results clearly show that OI significantly attenuated the production of LPS-induced pro-inflammatory cytokines in THP-1 human macrophages. Notably, LPS and/or OI treatment did not affect THP-1 cell survival; the trypan blue ratio (Fig. 2G) and LDH release level (Fig. $2 \mathrm{H})$ were unchanged after LPS and/or OI treatment. As expected, high basal levels of TNF- $\alpha$, IL-1 $\beta$, and IL- 6 were detected in the medium of ex vivo cultured SLE patient-derived PBMCs (Fig. 2I-K). Treatment with OI $(25 \mu \mathrm{M})$ significantly inhibited the production of TNF- $\alpha$, IL-1 $\beta$, and IL-6 in the patient-derived PBMCs (Fig. 2I-K). The applied OI treatment did not induce significant cell death in PBMCs (Fig. 2L). Thus, OI potently inhibited the production of proinflammatory cytokines in LPS-treated THP-1 cells and SLE patient-derived PBMCs.
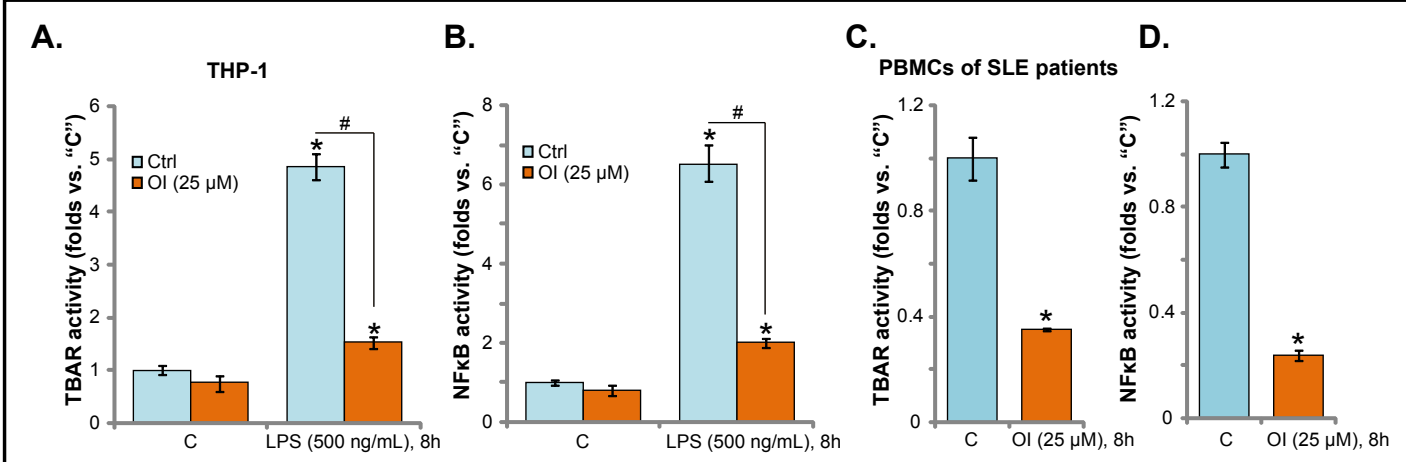

Fig. 3. OI inhibited lipid peroxidation and NF- $\kappa B$ activation in LPS-treated THP-1 cells and SLE patientderived PBMCs. THP-1 cells were pre-treated with OI) $(25 \mu \mathrm{M})$ for $60 \mathrm{~min}$, followed by LPS (500 $\mathrm{ng} / \mathrm{mL})$ treatment for the indicated times. Lipid peroxidation and NF- $\kappa B$ activation were tested with the TBAR activity assay (A) and p65 DNA-binding assay (B), respectively. SLE patient-derived PBMCs were treated with OI (25 $\mu \mathrm{M}$ ) for the indicated times. Lipid peroxidation (C) and NF- $\kappa B$ activation (D) were tested similarly. Data are presented as the mean \pm standard deviation. Assays A and B were replicated five times (five replicate well/ dishes). $C$ and D show the integrated results of PBMCs from all SLE patients $(n=5)$. Ctrl indicates vehicle control. $C$ indicates untreated control cells. ${ }^{*} \mathrm{p}<0.05$ vs. $C$ treatment. ${ }^{*} \mathrm{p}<0.05$. The experiments in this figure were repeated three times, and each time the results were similar. 
A.

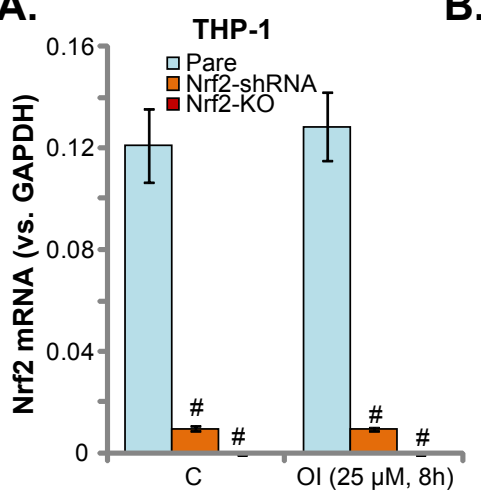

B.

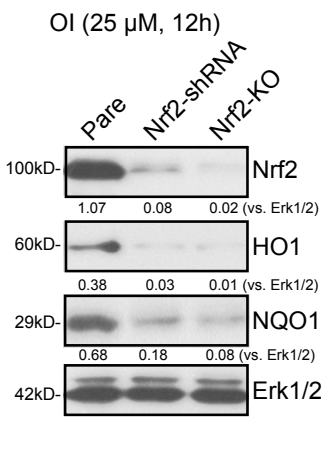

C.

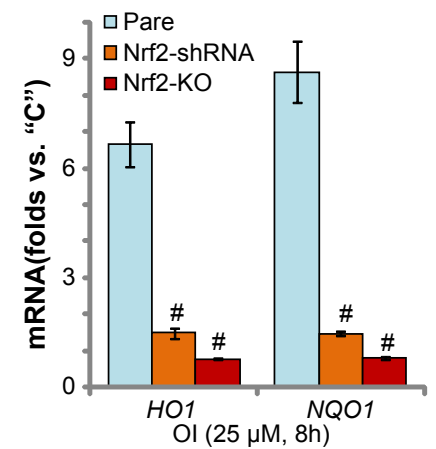

E.

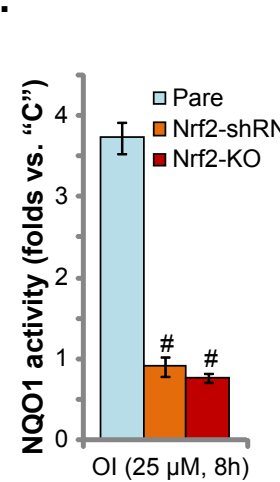

G.

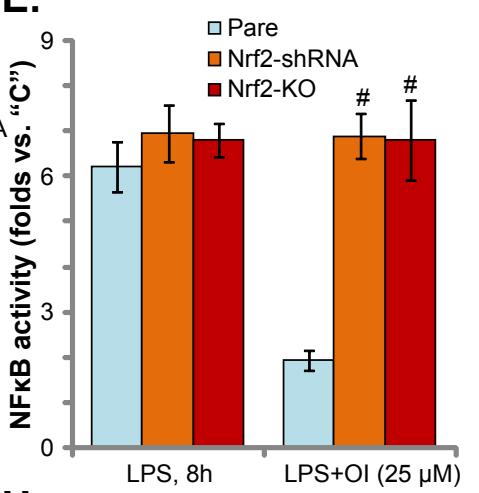

$\mathrm{H}$.

PBMCs of SLE patients

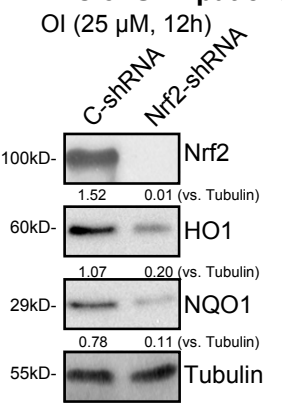

F. apare

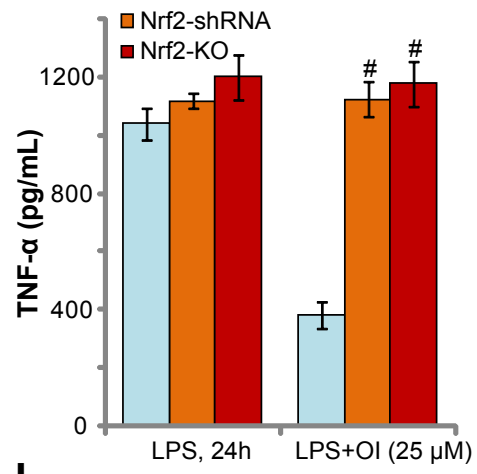

I.

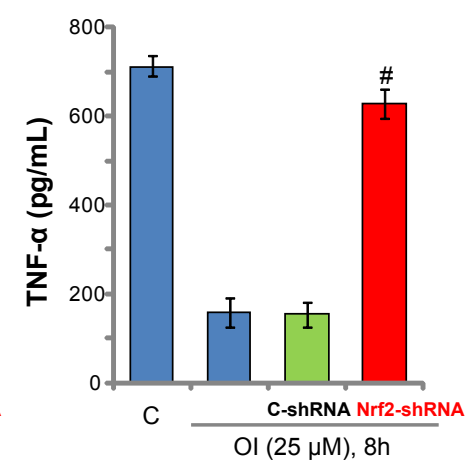

Fig. 4. Nrf2 activation was required for OI-induced anti-inflammatory actions in THP-1 human macrophages and SLE patient-derived PBMCs. Stable THP-1 cells with the lentiviral Nrf2 shRNA or the lentiCRISPRGFP-Nrf2 KO construct ("Nrf2-KO"), as well as the parental control cells (Pare), were treated with OI (25 $\mu \mathrm{M}$ ) for the indicated times. mRNA and protein expression levels of the listed genes were tested by qPCR assay and Western blotting (A-C); Relative NQ01 activity was also tested (D). Cells were also treated with LPS (500 ng/mL) for the indicated times. NF- $\kappa B$ activation and TNF- $\alpha$ production were tested by the p65 DNA-binding assay (E) and ELISA assay (F), respectively. SLE patient-derived PBMCs, with Nrf2 shRNA or scramble control shRNA (C-shRNA), were treated with OI $(25 \mu \mathrm{M})$ for the indicated times. The expression of the listed proteins was tested by Western blotting $(\mathrm{G})$. NFKB activation $(\mathrm{H})$ and TNF- $\alpha$ production (I) were also examined. The expression of the listed proteins was quantified and normalized to the loading control (B and G). Data are presented as the mean \pm standard deviation. In A-F each assay was repeated five times (five replicate well/dishes). Integrated results in G-I of PBMCs from all SLE patients ( $\mathrm{n}=5)$.C indicates untreated control cells. ${ }^{\#} \mathrm{p}<0.05$. vs. Pare cells $(\mathrm{A}-\mathrm{F}) .{ }^{\#} \mathrm{p}<0.05$. vs. C-shRNA cells (G-I). The experiments in this figurewere repeated three times, and each time the results were similar. 


\section{\begin{tabular}{ll} 
Cellular Physiology & Cell Physiol Biochem 2018;51:979-990 \\
\hline DOl: 10.1159/000495400 & O 2018 The Author(s). Published by S. Karger AG, Basel
\end{tabular}

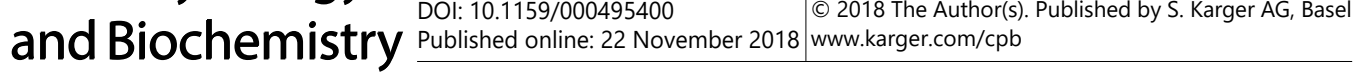 \\ Tang et al.: 4-Octyl Itaconate Activates Nrf2 Signaling in Monocytes}

OI inhibited lipid peroxidation and NF- $K B$ activation in LPS-treated THP-1 cells and SLE patient-derived PBMCs

LPS stimulation is known to induce the production of reactive oxygen species (ROS), which is essential for downstream NF- $\kappa$ B activation and the production of pro-inflammatory cytokines $[34,35]$. The TBARS level was examined to reflect cellular lipid peroxidation, a key cellular event following oxidative stress. Our results show that OI suppressed lipid peroxidation in LPS-treated THP-1 cells, verifying its anti-oxidant activity (Fig. 3A). Moreover, LPS-induced NFKB activation, tested by a p65 DNA-binding activity assay, was dramatically inhibited by OI pretreatment (Fig. 3B). In the SLE patient-derived PBMCs, OI treatment (25 $\mu \mathrm{M}$ ) similarly inhibited lipid peroxidation (Fig. 3C) and NFKB activation (Fig. 3D).

Nrf2 activation is required for OI-induced anti-inflammatory actions in THP-1 human macrophages and SLE patient-derived PBMCS

To confirm that Nrf2 activation is required for OI-induced anti-inflammatory actions, the shRNA strategy was utilized to knockdown Nrf2 in THP-1 human macrophages. Lentiviral human Nrf2 shRNA was transfected to THP-1 cells, and the stable cells were selected with puromycin. Furthermore, a lentiCRISPR-GFP-Nrf2 KO construct (from Dr. Li [30]) was applied to completely knockout Nrf2 in THP-1 cells. As shown, the expression level of Nrf2 mRNA was significantly reduced in stable THP-1 cells with Nrf2 shRNA or the Nrf2-KO construct (Fig. 4A). OI-induced Nrf2 protein stabilization (Fig. 4A), H01/NQ01mRNA (Fig. 4C), and protein (Fig. 4B) expression, as well as NQO1 activation (Fig. 4D), were almost blocked by Nrf2 silencing or KO (Fig. 4B-D). Importantly, OI pre-treatment did not inhibit LPS-induced NFKB activation (Fig. 4E) and TNF- $\alpha$ production (Fig. 4F) in Nrf2-silenced or -KO THP-1 cells.

In SLE patient-derived PBMCs, the addition of the lentiviral human Nrf2 shRNA also reversed the accumulation of OI-induced Nrf2 protein (Fig. 4G) and H01/NQO1 protein expression (Fig. 4G). OI-induced inhibition of NFKB activation (Fig. 4H) and TNF- $\alpha$ production (Fig. 4I) were almost completely reversed by Nrf2 shRNA. The scramble control shRNA was ineffective (Fig. 4G-I). These results together suggest that Nrf2 activation is required for OIinduced anti-inflammatory activity in THP-1 human macrophages and SLE patient-derived PBMCs.

\section{Discussion}

Imbalances between pro- and anti-inflammatory cytokines play an essential role in the pathogenesis of SLE $[1,3,5,36]$. Studies have shown that several key pro-inflammatory cytokines, including TNF- $\alpha$, IL- $1 \beta$, and IL- 6 , are significantly increased in the serum of SLE patients $[4,5]$. Elevations of these pro-inflammatory cytokines correlate with disease progression $[1,3,5,36]$. More importantly, TNF- $\alpha$, IL-6, and IL-10 levels are dramatically elevated in SLE patients with lupus nephritis [5-7, 36]. Conversely, the inhibition of proinflammatory cytokines could significantly slow the progression of SLE $[1,3,5,36]$. It has been previously shown that SLE patients with anti-TNF- $\alpha$ monoclonal antibody treatment show improvement in disease activity [9]. Furthermore, the blocking of IL-1 $\beta$ signaling in SLE patients could also be beneficial for lupus-related manifestations [7]. Additionally, antiIL-6 receptor antibody treatment in SLE patients induces a significant clinical improvement in SLE patients with arthritis [8].

Ye et al. showed that the expression levels of TNF- $\alpha$, IL-1 $\beta$, and IL- 6 are significantly increased in PBMCs of SLE patients compared with healthy controls [37]. ROS production and oxidative stress play essential roles in the activation of NF-kB signaling and the production of pro-inflammatory cytokines in SLE progression [38]. Conversely, ROS scavengers and anti-oxidant agents can attenuate inflammatory responses [38]. Activation of Nrf2 could efficiently inhibit ROS production and subsequently inhibit the production of NFKB-mediated pro-inflammatory cytokines [38]. In the current study, we showed that OI, the cell-permeable derivative of itaconate, induced Keap1-Nrf2 disassociation, Nrf2 protein accumulation, and 


\section{Cellular Physiology Cell Physiol Biochem 2018;51:979-990

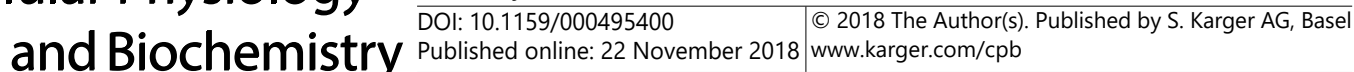 \\ Tang et al.: 4-Octyl Itaconate Activates Nrf2 Signaling in Monocytes}

nuclear translocation and activation in THP-1 human macrophages. Similarly, OI induced profound Nrf2 activation in SLE patient-derived PBMCs. In both LPS-activated THP-1 cells and SLE patient-derived PBMCs, OI treatment significantly inhibited mRNA expression and the production of multiple key pro-inflammatory cytokines (TNF- $\alpha$, IL-1 $\beta$, and IL-6). Further studies showed that OI potently inhibited NF- $\kappa B$ activation in the PBMCs and LPS-activated THP-1 cells.

Our results further showed that Nrf2 activation is required for OI-induced antioxidant and anti-inflammatory activities. Nrf2 silencing (by targeted shRNA) or KO (by CRISPR/ Cas9 gene-editing method) almost completely abolished OI-induced actions in SLE patientderived PBMCs and LPS-activated THP-1 cells. Compared with the other Nrf2 activators, one key advantage of this novel compound is that it is directly associated with Keap1 and causes Keap1 acetylation and disassociation from Nrf2. This should lead to direct and sustained Nrf2 activation.

\section{Conclusion}

Our results showed that OI activated Nrf2 signaling to potently inhibit the production of pro-inflammatory cytokines in SLE patient-derived PBMCs and human macrophages. Thus, OI and itaconate could have important therapeutic value for the future treatment of SLE.

\section{Acknowledgements}

This work was generously supported by the Medical Scientific Research Foundation of Guangdong Province of China (A2018071) and the Natural Science Foundation of Guangdong Province of China (2014A030313009).

\section{Disclosure Statement}

The authors have no conflicts of interest to declare.

\section{References}

1 Mohan C, Putterman C: Genetics and pathogenesis of systemic lupus erythematosus and lupus nephritis. Nat Rev Nephrol 2015;11:329-341.

2 Tsokos GC, Lo MS, Costa Reis P, Sullivan KE: New insights into the immunopathogenesis of systemic lupus erythematosus. Nat Rev Rheumatol 2016;12:716-730.

-3 Morel L: Immunometabolism in systemic lupus erythematosus. Nat Rev Rheumatol 2017;13:280-290.

4 Bezalel S, Asher I, Elbirt D, Sthoeger ZM: Novel biological treatments for systemic lupus erythematosus: current and future modalities. Isr Med Assoc J 2012;14:508-514.

-5 Su DL, Lu ZM, Shen MN, Li X, Sun LY: Roles of pro- and anti-inflammatory cytokines in the pathogenesis of SLE. J Biomed Biotechnol 2012;2012:347141.

-6 Herrera-Esparza R, Barbosa-Cisneros 0, Villalobos-Hurtado R, Avalos-Diaz E: Renal expression of IL-6 and TNFalpha genes in lupus nephritis. Lupus 1998;7:154-158.

-7 Ostendorf B, Iking-Konert C, Kurz K, Jung G, Sander O, Schneider M: Preliminary results of safety and efficacy of the interleukin 1 receptor antagonist anakinra in patients with severe lupus arthritis. Ann Rheum Dis 2005;64:630-633.

8 Illei GG, Shirota Y, Yarboro CH, Daruwalla J, Tackey E, Takada K, Fleisher T, Balow JE, Lipsky PE: Tocilizumab in systemic lupus erythematosus: data on safety, preliminary efficacy, and impact on circulating plasma cells from an open-label phase I dosage-escalation study. Arthritis Rheum 2010;62:542-552. 


\section{Cellular Physiology Cell Physiol Biochem 2018;51:979-990 and Biochemistry \begin{tabular}{l|l|l}
$\overline{D O I}: 10.1159 / 000495400$ & C 2018 The Author(s). Published by S. Karger AG, Basel
\end{tabular}

-9 Aringer M, Smolen JS: Therapeutic blockade of TNF in patients with SLE-promising or crazy? Autoimmun Rev 2012;11:321-325.

10 Suzuki T, Yamamoto M: Molecular basis of the Keap1-Nrf2 system. Free Radic Biol Med 2015;88:93-100.

11 Li W, Kong AN: Molecular mechanisms of Nrf2-mediated antioxidant response. Mol Carcinog 2009;48:91104.

12 Itoh K, Tong KI, Yamamoto M: Molecular mechanism activating Nrf2-Keap1 pathway in regulation of adaptive response to electrophiles. Free Radic Biol Med 2004;36:1208-1213.

13 Yoh K, Itoh K, Enomoto A, Hirayama A, Yamaguchi N, Kobayashi M, Morito N, Koyama A, Yamamoto M, Takahashi S: Nrf2-deficient female mice develop lupus-like autoimmune nephritis. Kidney Int 2001;60:1343-1353.

14 Wardyn JD, Ponsford AH, Sanderson CM: Dissecting molecular cross-talk between Nrf2 and NF-kappaB response pathways. Biochem Soc Trans 2015;43:621-626.

15 Kundu JK, Surh YJ: Nrf2-Keap1 signaling as a potential target for chemoprevention of inflammationassociated carcinogenesis. Pharm Res 2010;27:999-1013.

16 Mills EL, Ryan DG, Prag HA, Dikovskaya D, Menon D, Zaslona Z, Jedrychowski MP, Costa ASH, Higgins M, Hams E, Szpyt J, Runtsch MC, King MS, McGouran JF, Fischer R, Kessler BM, McGettrick AF, Hughes MM, Carroll RG, Booty LM, Knatko EV, Meakin PJ, Ashford MLJ, Modis LK, Brunori G, Sevin DC, Fallon PG, Caldwell ST, Kunji ERS, Chouchani ET, Frezza C, Dinkova-Kostova AT, Hartley RC, Murphy MP, O’Neill LA: Itaconate is an anti-inflammatory metabolite that activates Nrf2 via alkylation of KEAP1. Nature 2018;556:113-117.

17 Wang QM, Wang H, Li YF, Xie ZY, Ma Y, Yan JJ, Gao YF, Wang ZM, Wang LS: Inhibition of EMMPRIN and MMP-9 Expression by Epigallocatechin-3-Gallate through 67-kDa Laminin Receptor in PMA-Induced Macrophages. Cell Physiol Biochem 2016;39:2308-2319.

-18 Sindhu S, Al-Roub A, Koshy M, Thomas R, Ahmad R: Palmitate-Induced MMP-9 Expression in the Human Monocytic Cells is Mediated through the TLR4-MyD88 Dependent Mechanism. Cell Physiol Biochem 2016;39:889-900.

19 Ouyang Q, Wu J, Jiang Z, Zhao J, Wang R, Lou A, Zhu D, Shi GP, Yang M: Microarray Expression Profile of Circular RNAs in Peripheral Blood Mononuclear Cells from Rheumatoid Arthritis Patients. Cell Physiol Biochem 2017;42:651-659.

20 Du SL, Yuan X, Zhan S, Tang LJ, Tong CY: Trametinib, a novel MEK kinase inhibitor, suppresses lipopolysaccharide-induced tumor necrosis factor (TNF)-alpha production and endotoxin shock. Biochem Biophys Res Commun 2015;458:667-673.

21 Chanput W, Mes J, Vreeburg RA, Savelkoul HF, Wichers HJ: Transcription profiles of LPS-stimulated THP-1 monocytes and macrophages: a tool to study inflammation modulating effects of food-derived compounds. Food Funct 2010;1:254-261.

22 Li C, Yan K, Wang W, Bai Q, Dai C, Li X, Huang D: MIND4-17 protects retinal pigment epithelium cells and retinal ganglion cells from UV. Oncotarget 2017;8:89793-89801.

-23 Wu T, Li J, Li Y, Song H: Antioxidant and Hepatoprotective Effect of Swertiamarin on Carbon TetrachlorideInduced Hepatotoxicity via the Nrf2/HO-1 Pathway. Cell Physiol Biochem 2017;41:2242-2254.

-24 Sato AY, Tu X, McAndrews KA, Plotkin LI, Bellido T: Prevention of glucocorticoid induced-apoptosis of osteoblasts and osteocytes by protecting against endoplasmic reticulum (ER) stress in vitro and in vivo in female mice. Bone 2015;73C:60-68.

25 Ji F, Mao L, Liu Y, Cao X, Xie Y, Wang S, Fei H: K6PC-5, a novel sphingosine kinase 1 (SphK1) activator, alleviates dexamethasone-induced damages to osteoblasts through activating SphK1-Akt signaling. Biochem Biophys Res Commun 2015;458:568-575.

26 Xie J, Li Q, Ding X, Gao Y: GSK1059615 kills head and neck squamous cell carcinoma cells possibly via activating mitochondrial programmed necrosis pathway. Oncotarget 2017;10.18632/oncotarget.15135

-27 Tang XF, Liu HY, Wu L, Li MH, Li SP, Xu HB: Ginseng Rh2 protects endometrial cells from oxygen glucose deprivation/re-oxygenation. Oncotarget 2017;8:105703-105713.

28 Cortizo AM, Bruzzone L, Molinuevo S, Etcheverry SB: A possible role of oxidative stress in the vanadiuminduced cytotoxicity in the MC3T3E1 osteoblast and UMR106 osteosarcoma cell lines. Toxicology 2000;147:89-99.

-29 Hu H, Hao L, Tang C, Zhu Y, Jiang Q, Yao J: Activation of KGFR-Akt-mTOR-Nrf2 signaling protects human retinal pigment epithelium cells from Ultra-violet. Biochem Biophys Res Commun 2018;495:2171-2177. 


\section{Cellular Physiology Cell Physiol Biochem 2018;51:979-990

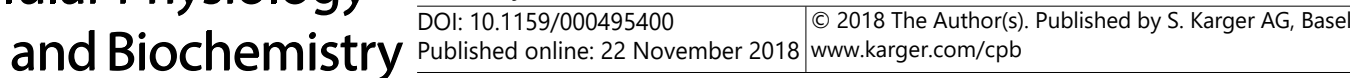 \\ Tang et al.: 4-Octyl Itaconate Activates Nrf2 Signaling in Monocytes}

30 Shi X, Liu HY, Li SP, Xu HB: Keratinocyte growth factor protects endometrial cells from oxygen glucose deprivation/re-oxygenation via activating Nrf2 signaling. Biochem Biophys Res Commun 2018;501:178185.

-31 Ito M, Yamamoto T, Watanabe M, Ihara T, Kamiya H, Sakurai M: Detection of measles virus-induced apoptosis of human monocytic cell line (THP-1) by DNA fragmentation ELISA. FEMS Immunol Med Microbiol 1996;15:115-122.

-32 Li P, Fan JB, Gao Y, Zhang M, Zhang L, Yang N, Zhao X: miR-135b-5p inhibits LPS-induced TNFalpha production via silencing AMPK phosphatase Ppm1e. Oncotarget 2016;7:77978-77986.

33 Zhang H, Davies KJ, Forman HJ: Oxidative stress response and Nrf2 signaling in aging. Free Radic Biol Med 2015;88:314-336.

-34 Sanlioglu S, Williams CM, Samavati L, Butler NS, Wang G, McCray PB, Jr., Ritchie TC, Hunninghake GW, Zandi E, Engelhardt JF: Lipopolysaccharide induces Rac1-dependent reactive oxygen species formation and coordinates tumor necrosis factor-alpha secretion through IKK regulation of NF-kappa B. J Biol Chem 2001;276:30188-30198.

-35 Park HS, Jung HY, Park EY, Kim J, Lee WJ, Bae YS: Cutting edge: direct interaction of TLR4 with NAD(P)H oxidase 4 isozyme is essential for lipopolysaccharide-induced production of reactive oxygen species and activation of NF-kappa B. J Immunol 2004;173:3589-3593.

-36 Yu SL, Kuan WP, Wong CK, Li EK, Tam LS: Immunopathological roles of cytokines, chemokines, signaling molecules, and pattern-recognition receptors in systemic lupus erythematosus. Clin Dev Immunol 2012;2012:715190.

37 Ye L, Ji L, Wen Z, Zhou Y, Hu D, Li Y, Yu T, Chen B, Zhang J, Ding L, Du J, Huang Z: IL-37 inhibits the production of inflammatory cytokines in peripheral blood mononuclear cells of patients with systemic lupus erythematosus: its correlation with disease activity. J Transl Med 2014;12:69.

-38 Jiang T, Tian F, Zheng H, Whitman SA, Lin Y, Zhang Z, Zhang N, Zhang DD: Nrf2 suppresses lupus nephritis through inhibition of oxidative injury and the NF-kappaB-mediated inflammatory response. Kidney Int 2014;85:333-343. 